\title{
Temperature and atmospheric pressure may be considered as predictors for the occurrence of bacillary dysentery in Guangzhou, Southern China
}

\author{
Tiegang $L^{[1]}$, Zhicong Yang ${ }^{[1]}$ and Ming Wang ${ }^{[1]}$
}

[1]. Guangzhou Center for Disease Control and Prevention, Guangzhou, PR, China.

\begin{abstract}
Introduction: The control of bacillary dysentery (BD) remains a big challenge for China. Methods: Negative binomial multivariable regression was used to study relationships between meteorological variables and the occurrence of $\mathrm{BD}$ during the period of 2006-2012. Results: Each $1^{\circ} \mathrm{C}$ rise of temperature corresponded to an increase of $3.60 \%(95 \% \mathrm{CI}, 3.03 \%$ to $4.18 \%)$ in the monthly number of BD cases, whereas a $1 \mathrm{hPa}$ rise in atmospheric pressure corresponded to a decrease in the number of $\mathrm{BD}$ cases by $2.85 \%(95 \% \mathrm{CI}=3.34 \%$ to $2.37 \%$ decrease $)$. Conclusions: Temperature and atmospheric pressure may be considered as predictors for the occurrence of BD in Guangzhou.
\end{abstract}

Keywords: Bacillary dysentery. Weather factors. Correlation.

Bacillary dysentery (BD), also known as shigellosis, is a common intestinal infectious disease caused by Shigella. Worldwide, the incidence of $\mathrm{BD}$ is estimated to be 164.7 million cases per year, 163.2 million of which were in developing countries, where 1.1 million deaths occurred ${ }^{1}$. As the largest trading city in southern China, Guangzhou is a representative city with a high prevalence of BD. It was reported that, during the period of 1997-2005, the annual incidence rate of BD ranged from 15.24 to 20.50 per 100,000 inhabitants $^{2}$, raising concerns among public health authorities regarding its high incidence.

BD control is a serious challenge due to many factors. Currently, one of the critical programs for preventing BD is concentrated on monitoring and predicting BD incidence. Although previous studies have revealed significant correlations between meteorological factors and $\mathrm{BD}$ infection ${ }^{3}$, the influence of these variables depends on differences in socioeconomic backgrounds and climate patterns in different regions $s^{4,5}$. Moreover, in southern China, very little information is available regarding the use of meteorological variables as predictors for the occurrence and transmission of BD. The goals of this study were to examine the effects of weather patterns on the incidence of BD in Guangzhou between 2006 and 2012 and to develop a simple, precise, and low-cost functional early warning system.

We obtained BD incidence data for Guangzhou from the National Notifiable Disease Report System (NNDRS).

Address to: Dr. Tiegang Li. Guangzhou Center for Disease Control and Prevention. Qide Rd, n. 1, Jiahe, Baiyun, Guangzhou, 510440 Guangdong Province, China.

Phone: 8620 3605-5887; Fax: 8620 3605-5885

e-mail: Tiegang1977@126.com

Received 17 July 2013

Accepted 22 January 2014
In China, BD is a notifiable Class-B communicable disease, and all cases are diagnosed according to the unified diagnostic criteria issued by the Chinese Ministry of Health. For a patient's illness to meet the case definition for BD clinical symptoms (e.g., diarrhea, fever, abdominal pains, tenesmus, and stool mixed with blood), samples should be taken for laboratory confirmation (e.g., dysentery bacilli identification and genetic material detection). A standard form was adopted by local physicians and epidemiologists to collect information from infected individuals. Routine case reporting is done by hospitals through the NNDRS within 24 hours. Simultaneous meteorological data, including daily average temperature (in ${ }^{\circ} \mathrm{C}$ ), relative humidity $(\%)$, atmospheric pressure (in $\mathrm{hPa}$ ), wind velocity (in $\mathrm{m} / \mathrm{s}$ ), duration of sunshine (in $\mathrm{h} /$ day) and degree of rainfall (in $\mathrm{mm}$ ) were obtained from documentation by the Guangzhou Meteorological Bureau.

A negative binomial regression was used to study relationships between meteorological variables and $\mathrm{BD}$ incidence. A negative binomial distribution is a Poisson distribution with an extra dispersion term. The extra dispersion term acts as a random effect that subjects the Poisson means to additional variation that has a gamma distribution. Given the data were over-dispersed; we chose a negative binomial distribution model rather than a Poison model. The cases were BD occurrence. Data are presented as the prevalence of BD per 100,000 inhabitants, grouped by month of onset and for each meteorological variable, with a monthly average or aggregate being calculated. A preliminary analysis was conducted using a Pearson's correlation coefficient (' $r$ ') matrix with meteorological variables. This indicated that the model constructed using contemporaneously both temperature and atmospheric pressure suffered from collinearity problems, because the 2 variables showed a strong negative correlation $(\mathrm{r}=-0.86, \mathrm{p}<0.01$; Table 1). Thus, we tested 2 separate 
TABLE 1 - Pearson's correlation coefficient (' $r$ ') matrix of meteorological variables in Guangzhou, southern China, $2006-2012$.

\begin{tabular}{|c|c|c|c|c|c|c|}
\hline & $\begin{array}{c}\text { Atmospheric } \\
\text { pressure }\end{array}$ & $\begin{array}{l}\text { Relative } \\
\text { humidity }\end{array}$ & $\begin{array}{c}\text { Average } \\
\text { temperature }\end{array}$ & Rainfall & Sunshine & $\begin{array}{c}\text { Wind } \\
\text { velocity }\end{array}$ \\
\hline Atmospheric pressure & 1.00 & & & & & \\
\hline Average temperature & $-0.86(\mathrm{p}=0.00)$ & $0.32(\mathrm{p}=0.00)$ & 1.00 & & & \\
\hline Rainfall & $-0.58(\mathrm{p}=0.00)$ & $0.52(\mathrm{p}=0.00)$ & $0.53(\mathrm{p}=0.00)$ & 1.00 & & \\
\hline Wind velocity & $-0.10(\mathrm{p}=0.36)$ & $0.20(\mathrm{p}=0.06)$ & $-0.28(\mathrm{p}=0.01)$ & $-0.12(\mathrm{p}=0.29)$ & $0.00(\mathrm{p}=0.99)$ & 1.00 \\
\hline
\end{tabular}

TABLE 2 - Negative binomial regression model of meteorological factors associated with risk of bacillary dysentery incidence*.

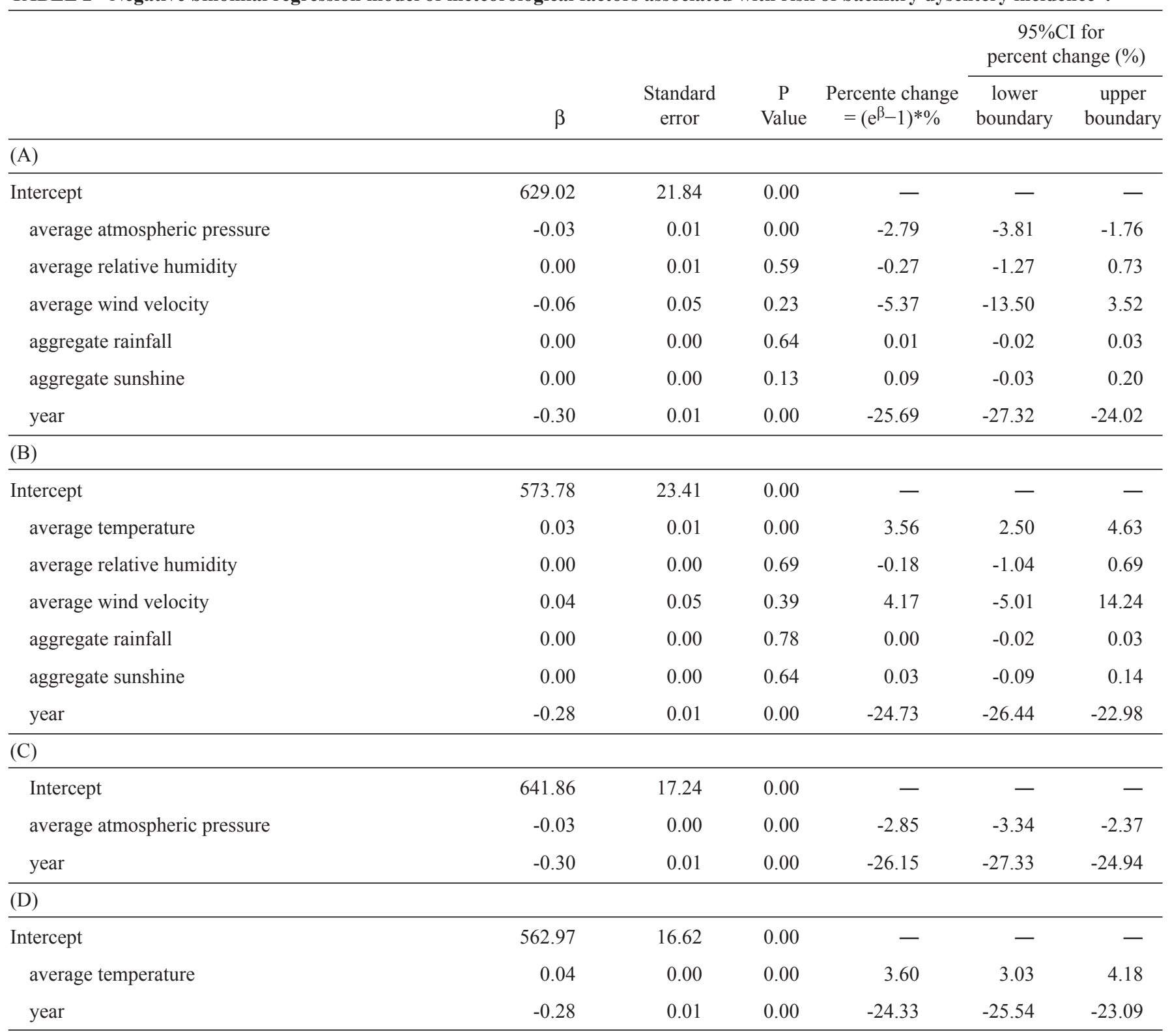

95\%CI: 95\% confidence interval. *Negative binomial regression model for monthly BD incidences, not including (A) average temperature (A) or (B) atmospheric pressure (B). Final models, not including (C) average temperature or (D) atmospheric pressure. 
negative binomial regression models: the first included average temperatures, but no atmospheric pressure data, while the second considered atmospheric pressure, but not temperature data. Both models also included relative humidity, wind velocity, duration of sunshine, the degree of rainfall, and the year as independent variables. The final model included only those variables that reached a $p$ value of $<0.05$ in the preliminary model with all variables included. To quantify the effects of meteorological variables, we computed the influences $\left(\mathrm{e}^{\beta}-1\right)$, which virtually correspond to the percent change. These analyses were carried out using statistical analysis software (SAS) (V.8.01, SAS Institute, Cary, New Jersey, USA).

From January 1, 2006 to December 31, 2012, 4775 confirmed BD cases were reported in Guangzhou, of which $55.44 \%(2,481)$ were male patients and $44.56 \%$ (1994) were female patients. The proportion of confirmed cases in age ranges between $\leq 5,6-19,20-44,45-64$, and $\geq 65$ was $36.08 \%(1,723), 7.48 \%$ (357), 35.48\% (1,694), 13.24\% (632), and 7.73\% (369), respectively. Of the 6 meteorological variables studied, temperature and atmospheric pressure were statistically significant in the final model (both $\mathrm{p}<0.01$ ). After adjusting by year, each $1{ }^{\circ} \mathrm{C}$ rise of temperature corresponded to an increase of $3.60 \%$ (95\% CI $3.03 \%$ to $4.18 \%$ ) in the monthly number of $\mathrm{BD}$ cases, whereas a $1 \mathrm{hPa}$ rise of atmospheric pressure corresponded to a decrease in the number of cases by $2.85 \%(95 \%$ CI $-3.34 \%$ to $-2.37 \%)$, causing a negative effect (Table 2). The residuals plots indicated that model residuals did not violate the statistical modeling assumptions.

Weather factors such as temperature and humidity have been proven to exert a significant influence on the occurrence and transmission of some infectious diseases. For example, in Switzerland, higher water vapor pressure and temperature were associated with increased risks for developing communityacquired Legionnaires' disease ${ }^{6}$. In Botswana, the elevation of annual minimum temperature was considered as a critical factor for the continual ascent in the number of diarrheal diseases reported during the period of 1974-20037. The result of current study revealed that temperature was positively correlated with $\mathrm{BD}$ incidences in Guangzhou, with each $1{ }^{\circ} \mathrm{C}$ rise in temperature corresponding to an increase of $3.60 \%$ in the monthly number of confirmed BD cases. This finding is in general agreement with previous findings ${ }^{3}$, which suggested that temperature may be considered a precipitating factor for $\mathrm{BD}$ incidence. In addition, we found that atmospheric pressure was negatively associated with BD incidence in the same month. A similar observation was also reported in northeast China ${ }^{8}$. The mechanism whereby climate affects the incidence of BD is still unclear. A possible explanation may be that meteorological factors may directly affect the replication of bacterial and protozoan pathogens and affect environmental reservoirs ${ }^{9,10}$.

Some limitations must be acknowledged for this study. For example, the incubation period of 1-3 days for every case cannot be determined exactly. We chose to use monthly aggregated data for BD cases and monthly average or aggregate meteorological data. The errors of these approximations, however, are likely to be random, suggesting that our risk estimates are reliable. Moreover, as this investigation is an ecological study, we cannot exclude the possibility that we could not identify or consider some potential confounding variables. Although we emphasize the impact of climate, other factors such as human dietary lifestyle, hygiene habits, and Shigella strain susceptibility to drugs should be addressed in further studies.

Taken together, despite these limitations, we report that weather factors have a significant influence on the occurrence and transmission of $\mathrm{BD}$. A rise of temperature may increase the risk of $\mathrm{BD}$ infection, whereas an increase in atmospheric pressure may reduce the risk of $\mathrm{BD}$ infection. These 2 meteorological variables may be considered as predictors for the occurrence of bacillary dysentery in Guangzhou.

\section{CONFLICT OF INTEREST}

The authors declare that there is no conflict of interest.

\section{REFERENCES}

1. World Health Organization. Shigellosis: disease burden, epidemiology and case management. Wkly Epidemiol Rec 2005; 80:94-99.

2. Wei YH, Yang ZC, Kang Y. 2006-2010 Guangzhou bacillary dysentery epidemic trend analysis, 2006-2010. [Article in Chinese] South China J Prev Med 2011; 37:61-63.

3. Ma W, Sun X, Song Y, Tao F, Feng W, He Y, et al. Applied mixed generalized additive model to assess the effect of temperature on the incidence of bacillary dysentery and its forecast. PLoS One 2013; 8:e62122.

4. Zhang Y, Bi P, Hiller JE. Weather and the transmission of bacillary dysentery in Jinan, northern China: a time-series analysis. Public Health Rep 2008; 123:61-66.

5. Ma SL, Tang QL, Liu HW, He J, Gao SH. Correlation analysis for the attack of bacillary dysentery and meteorological factors based on the Chinese medicine theory of Yunqi and the medical-meteorological forecast model. Chin J Integr Med 2013; 19:182-186.

6. Conza L, Casati S, Limoni C, Gaia V. Meteorological factors are associated with an increased risk of community-acquired Legionnaires' disease in Switzerland: an epidemiological study. BMJ Open 2013; 3:e002428.

7. Alexander KA, Carzolio M, Goodin D, Vance E. Climate Change is Likely to Worsen the Public Health Threat of Diarrheal Disease in Botswana. Int J Environ Res Public Health 2013; 10:1202-1230.

8. Huang D, Guan P, Guo J, Wang P, Zhou B. Investigating the effects of climate variations on bacillary dysentery incidence in northeast China using ridge regression and hierarchical cluster analysis. BMC Infect Dis 2008; 8:130.

9. Curriero FC, Patz JA, Rose JB, Lele S. The association between extreme precipitation and waterborne disease outbreaks in the United States, 19481994. Am J Public Health 2001; 91:1194-1199.

10. Lama JR, Seas CR, León-Barúa R, Gotuzzo E, Sack RB. Environmental temperature, cholera, and acute diarrhoea in adults in Lima, Peru. J Health Popul Nutr 2004; 22:399-403. 